\title{
Correspondence
}

\section{Intra-articular injection of phenylbutazone in gonarthrosis}

SIR, Between 1970 and 1986 we treated 15 patients suffering from severe chronic osteoarthritis of the knee with intra-articular injections of phenylbutazone. All were seen in our outpatient clinic with severely painful knees with effusion. Blood tests, synovial fluid analysis, including polarised light microscopic examination for crystals, and $x$ rays confirmed the clinical diagnosis of osteoarthritis.

They were treated successively with aspirin nonsteroidal anti-inflammatory drugs, physiotherapy, and intra-articular injections of steroids, all without success.

Intra-articular injections at first were given monthly, injecting $5 \mathrm{mg}$ dexamethasone or $20 \mathrm{mg}$ triamcinalone after draining the joint. As there was no improvement after three injections we gave three more injections twice monthly, again without success. The treatment was therefore continued with weekly injections during the three following months.

Despite these 16 injections 15 patients still had a painful swollen knee. These patients were offered the choice of continuing with the steroid injections (though we were reluctant to do so because of the possible side effects) or having injections of an anti-inflammatory drug in the hope of 'drying up' the effusion. They all volunteered to try the latter procedure.

Again the knee was aspirated, but care was taken to leave about $10 \mathrm{ml}$ synovial fluid in the joint so that the injected drugs would be diluted. We injected $1 \mathrm{ml}$ phenylbutazone $(190 \mathrm{mg})$ mixed with $5 \mathrm{ml}$ procaine $1 \%$.

Among the 15 patients, three experienced no pain at injection, but 12 declared the procedure moderately painful. Pain lasted for about one hour.

The patients returned for a check after one week. All were improved but not cured. Four required one more injection and 11 patients two more injections. In all cases effusion and pain subsided. Follow up has lasted from a few months to 10 years with no reappearance of the symptoms. Unfortunately we did not obtain synovial biopsy specimens.

In view of the good clinical results we believe that intraarticular injections of phenylbutazone in severe knee osteoarthritis with effusion is a helpful procedure.

We were unable to find any previous report on this subject, but Sárközi and Bartosiewicz reported a case of chondrocalcinosis treated successfully with this procedure. $^{1}$

Centre Médico-Social de Bruxelles, 69 rue des Chartreux,

B MOENS

Brussels,

Belgium

\section{References}

1 Sárközi A M, Bartosiewicz G. Intra-artikulaere Behandlung der Pseudogicht mit einem Mukopolysaccharidpolyschwefelsaeureester. Therapiewoche 1978; 28: 6294-9. CHR MOENS

\section{Nutritional status in patients with rheumatoid arthritis}

SIR, For some years we have had the impression that patients with rheumatoid arthritis in the UK were less well os nourished and had more severe disease than their Cana- $\overrightarrow{0}$ dian counterparts. The report by Helliwell et al seemed to support this belief by showing that $26 \%$ of 50 patients had $\vec{\omega}$ evidence of malnutrition and that this correlated with disease severity. ${ }^{1}$ We have assessed the nutritional status of 30 sequential patients with rheumatoid arthritis attending our outpatient department and of 30 subjects with osteoarthrosis or other non-inflammatory musculoligamentous disorders. All patients were under 65 years of $\infty$ age and none were taking systemic steroids. The measurements made were (a) clinical: $(i)$ skin fold thickness measured over the triceps and subscapular regions-the percentile of the sum thickness with respect to age and sex $\vec{\sim}$ was calculated from previously published tables; ${ }^{2}$ (ii) body $\overparen{\Phi}$ frame size was first calculated from the height and wrist circumference and using this calculation the percentage $\stackrel{\overparen{D}}{\rightrightarrows}$ ideal body weight was obtained from tables; ${ }^{2}(b)$ biochemical-serum albumin, prealbumin, and transferrin were measured by routine laboratory techniques.

Table 1 Clinical and biochemical parameters of the patients with osteoarthritis $(O A)$ or rheumatoid arthritis (RA) and of controls

\begin{tabular}{llll}
\hline & $O A$ & $R A$ & $p^{*}$ \\
\hline No (male/female) & $16 / 14$ & $13 / 17$ & $\mathrm{NS}$ \\
Average age (years) & 50 & 54 & $\mathrm{NS}$ \\
Ideal body weight (\%) & $115.6(14.5) \dagger$ & $116(18.5)$ & $\mathrm{NS}$ \\
Percentile for & & & \\
$\quad$ skin fold thickness & $54.8(29.3)$ & $59.5(26.4)$ & $\mathrm{NS}$ \\
Albumin (g/l) & $37.6(4.7)$ & $33.3(3.4)$ & $<0.001$ \\
Transferrin (g/l) & $3.19(0.42)$ & $3.01(0.47)$ & $\mathrm{NS}$ \\
Prealbumin (g/l) & $0.25(0.046)$ & $0.22(0.052)$ & $<0.02$
\end{tabular}

*p Values were calculated with an unpaired Student's $t$ test, uncorrected for the number of comparisons made.

†Values are mean (SD).

As can be seen from Table 1 the mean albumin levels were lower in patients with rheumatoid arthritis, but this is known, and correlate with disease activity. This is unlikely $\mathrm{N}$ to be a reflection of nutritional status. Only one patient $\omega$ with rheumatoid arthritis had an ideal body weight less $\sigma$ than $90 \%$, and one different patient fell below the 10 th percentile for skin fold thickness. Two patients with $\mathrm{OA}$ were under $90 \%$ of the ideal body weight, and three were $\mathscr{D}$ below the 10th percentile for skin fold thickness. These results do not suggest there is any major abnormality of 